\title{
Potential Errors in a Scheme of Universal Quantum Gates in Kane's Model
}

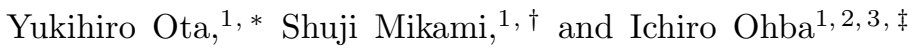 \\ ${ }^{1}$ Department of Physics, Waseda University, Tokyo 169-8555, Japan \\ ${ }^{2}$ Kagami Memorial Laboratory for Material Science and Technology, Waseda University, Tokyo 169-0051, Japan \\ ${ }^{3}$ Advanced Research Center for Science and Technology, Waseda University, Tokyo 169-8555, Japan
}

(Dated: December 16, 2018)

\begin{abstract}
We re-investigate a plausible proposal for universal quantum gates in Kane's model, in which the authors assumed that electron spin is always downward under a background magnetic field and the value of controlling parameters is varied instantaneously. We demonstrate that a considerable error appears, for example, in the X rotation. As result, the controlled operations don't work. Such a failure is caused by improper choice of the computational bases; actually, the electron spin is not always downward over time during quantum operations.
\end{abstract}

PACS numbers: 03.67.Lx

\section{INTRODUCTION}

Various physical models for quantum computation have been proposed. Among them, a nuclear magnetic resonance (NMR) quantum computation by liquid state NMR [1, 2, 3] is the most successful, because several quantum algorithms have experimentally worked, using five or seven qubits (see Ref. 4] for additional references). On the other hand, Gershenfeld and Chuang [2] pointed out that it was not possible to achieve beyond ten qubits using it.

Among many proposals for realizing quantum computation with many qubits [5, 6, 7], Kane [5] proposed quantum computing by nuclear spins in a semiconductor. In this proposal, the nuclear spin of a dopant atom, ${ }^{31} \mathrm{P}$, implanted into a silicon substrate is a single qubit, and quantum states are measured by detecting currents of spin-polarized electrons around ${ }^{31} \mathrm{P}$. Although the implantation of the atoms with requisite accuracy and the detection of the single electronic charge motion are very difficult even in the present state of the technology, Kane's proposal could achieve quantum computation by the use of many qubits, applying the several existing microfabrication techniques of semiconductors. Moreover, several important experimental techniques, for example, the single-ion implantation method [8] and the single-electron transistor 9, 10, 11, have been developed steadily. Hence, it is necessary to re-investigate the feasible conditions required by Kane's original proposal, based on detailed theoretical analyses and the present state of experimental techniques.

Several investigations of the schemes of implementing quantum gates in Kane's model have been reported. Wellard et al. 12] numerically derived an effective Hamiltonian and proposed a nonadiabatic controlling scheme for a controlled-NOT (СNOT) gate. It should be noted that

\footnotetext{
*Electronic address: oota@hep.phys.waseda.ac.jp

${ }^{\dagger}$ Electronic address: mikami@hep.phys.waseda.ac.jp

${ }^{\ddagger}$ Electronic address: ohba@waseda.jp
}

the quantum computational bases chosen are not eigenstates of their Hamiltonian. Fowler et al. 13] showed an adiabatic controlling scheme for a CNOT gate and studied the errors under the presence of dephasing. Their proposal is based on Goan and Milburn's study, which is explained briefly in Ref. [14]. Hill and Goan 15] proposed a nonadiabatic scheme for arbitrary single qubit operations and controlled operations (e.g., a CNOT gate, a swap gate, and a controlled $-\mathrm{Z}$ gate), and studied the effect of dephasing.

In particular, Hill and Goan's results are very important, as they make it possible to construct a set of universal gates for quantum computation [16]. They use the assumption that spin of the donor electrons is always downward, even if quantum gates are carried out. Furthermore, a nonadiabatic, or instantaneous, controlling process of parameters is an ideal one from both theoretical and experimental points of view. Therefore, it is necessary to discuss the validity of such assumption and idealization.

In this paper, we show that potential errors should exist in Hill and Goan's schemes for the gates including spin-flip operations (e.g., X rotations and CNOT gates); there is a large difference between their aimed state $\left|\phi_{\text {target }}\right\rangle$ obtained by quantum operations and the physical state $\left|\phi_{\text {phys }}\right\rangle$ obtained by a more realistic time evolution. We think that they didn't properly take account of the hyperfine interaction (HY) between electron spins and nuclear spins in their composition of the qubits system.

This paper is organized as follows. First, we review Kane's model and, taking the strengths of all interactions in the model into account, explain how to choose our computational bases in Sec.II After the completion of choosing them, we discuss the schemes of several important quantum gates, phase-shift operations, spin-flip operations, and controlled $-\mathrm{Z}$ operations in Sec.III The main results are shown in Sec rize our results in Sec.IV] 


\section{KANE'S MODEL}

Kane's model for two qubits is described by the Hamiltonian

$$
H(t)=\sum_{i=1}^{2} H^{i}(t)+J(t) \boldsymbol{\sigma}^{1 e} \cdot \boldsymbol{\sigma}^{2 e}+\sum_{i=1}^{2} H_{a c}^{i}(t),
$$

where $H^{i}(t)=-g_{n} \mu_{n} B \sigma_{z}^{i n}+\mu_{B} B \sigma_{z}^{i e}+A_{i}(t) \boldsymbol{\sigma}^{i e} \cdot \boldsymbol{\sigma}^{i n}$, $H_{a c}^{i}(t)=B_{a c} \boldsymbol{m}(t) \cdot\left(-g_{n} \mu_{n} \boldsymbol{\sigma}^{i n}+\mu_{B} \boldsymbol{\sigma}^{i e}\right)$ and $\boldsymbol{m}(t)=$ $\left(\cos \left(\omega_{a c} t\right),-\sin \left(\omega_{a c} t\right), 0\right)$. The Pauli matrix is written by $\sigma_{k}(k=x, y, z)$, and the superscript $i e(i n)$ in it indicates the electronic (nuclear) spin of the dopant atom implanted in the $i$ th site. According to Ref. [5], the value of the uniform static magnetic field in the direction of the $z$ axis is $2.0 \mathrm{~T}$ (i.e., $B=2.0 \mathrm{~T}$ ). Then, the value of the Zeeman splitting energies for the electron, $\mu_{B} B$, and the nucleus, $g_{n} \mu_{n} B$, are $0.116 \mathrm{meV}$ and $0.071 \times 10^{-3} \mathrm{meV}$, respectively. The HY on the $i$ th site between the nuclear spin and the electronic spin, $A_{i}(t) \boldsymbol{\sigma}^{i e} \cdot \boldsymbol{\sigma}^{i n}$ is locally changed by controlling the voltage in the $i$ th A-gate located right above the $i$ th dopant atom [5]. The typical value of the $\mathrm{HY}$ is $0.121 \times 10^{-3} \mathrm{meV}\left(\equiv A_{0}\right)$ when the voltage in the $\mathrm{A}$-gate is vanishing, and the magnitude of $A_{i}$ decreases as it increases [5]. Notice that the nuclear Zeemann splitting energy and the HY are almost the same order of magnitude. This causes a nonnegligible mixing between the electron spin down and up in the eigenstates of the Hamiltonian (see below). The electronic exchange interaction (EE) between the 1st and the 2nd electronic spins, $J(t) \boldsymbol{\sigma}^{1 e} \cdot \boldsymbol{\sigma}^{2 e}$, is locally changed by controlling the voltage in the $\mathrm{J}$-gate located between the neighboring dopant atoms [5]. We assume that it will be possible to change the magnitude of the EE from $J=0$ to $J \simeq \mu_{B} B / 2[15]$. The third term in Eq. (11) is a time-dependent interaction between the magnetic field perpendicular to the $z$ axis and spins. The typical value of $B_{a c}$ is assumed to be $B_{a c} \simeq 2.5 \mathrm{mT}[5,15]$. We write the eigenstates for $\sigma_{z}^{i n}$ as $|0\rangle_{i}$ and $|1\rangle_{i}\left(\sigma_{z}^{i n}|0\rangle_{i}=|0\rangle_{i}\right.$ and $\sigma_{z}^{i n}|1\rangle_{i}=-|1\rangle_{i}$ ), and those for $\sigma_{z}^{i e}$ as $|\uparrow\rangle_{i}$ and $|\downarrow\rangle_{i}$ $\left(\sigma_{z}^{i e}|\uparrow\rangle_{i}=|\uparrow\rangle_{i}\right.$ and $\left.\sigma_{z}^{i e}|\downarrow\rangle_{i}=-|\downarrow\rangle_{i}\right)$.

To investigate the time evolution of the system in detail, we first choose the states to represent a qubit from the eigenstates of $H^{i}(t)$ with a fixed value of $A_{i}(t)$. These eigenstates compose the temporal bases in the adiabatic calculation. We abbreviate the argument $t$ in the equations of its eigenvalues and eigenstates (we will recover it if necessary). Introducing $\epsilon=\mu_{B} B+g_{n} \mu_{n} B$, the eigenvalues $E_{k}^{i}\left(A_{i}\right)(k=0,1,2,3$ from the below) are given as follows:

$$
\begin{aligned}
& E_{0}^{i}\left(A_{i}\right)=-A_{i}-\sqrt{\epsilon^{2}+4 A_{i}^{2}} \\
& E_{1}^{i}\left(A_{i}\right)=-\mu_{B} B+g_{n} \mu_{n} B+A_{i}, \\
& E_{2}^{i}\left(A_{i}\right)=-A_{i}+\sqrt{\epsilon^{2}+4 A_{i}^{2}} \\
& E_{3}^{i}\left(A_{i}\right)=\mu_{B} B-g_{n} \mu_{n} B+A_{i} .
\end{aligned}
$$

Defining $\cos \theta_{i}=\left(\epsilon+\sqrt{\epsilon^{2}+4 A_{i}^{2}}\right) / N_{i}, \sin \theta_{i}=2 A_{i} / N_{i}$, and $N_{i}^{2}=2 \sqrt{\epsilon^{2}+4 A_{i}^{2}}\left(\epsilon+\sqrt{\epsilon^{2}+4 A_{i}^{2}}\right)$, their corresponding eigenstates $\left|u_{k}\left(A_{i}\right)\right\rangle_{i}$ are also given by

$$
\begin{aligned}
\left|u_{0}\left(A_{i}\right)\right\rangle_{i} & =-\sin \theta_{i}|\uparrow\rangle_{i}|1\rangle_{i}+\cos \theta_{i}|\downarrow\rangle_{i}|0\rangle_{i}, \\
\left|u_{1}\left(A_{i}\right)\right\rangle_{i} & =|\downarrow\rangle_{i}|1\rangle_{i} \\
\left|u_{2}\left(A_{i}\right)\right\rangle_{i} & =\cos \theta_{i}|\uparrow\rangle_{i}|1\rangle_{i}+\sin \theta_{i}|\downarrow\rangle_{i}|0\rangle_{i} \\
\left|u_{3}\left(A_{i}\right)\right\rangle_{i} & =|\uparrow\rangle_{i}|0\rangle_{i}
\end{aligned}
$$

The operator $S_{z}^{i} \equiv\left(\sigma_{z}^{i e}+\sigma_{z}^{i n}\right) / 2$ commutes with $H^{i}: \quad S_{z}^{i}\left|u_{0}\left(A_{i}\right)\right\rangle_{i}=S_{z}^{i}\left|u_{2}\left(A_{i}\right)\right\rangle_{i}=0, S_{z}^{i}\left|u_{1}\left(A_{i}\right)\right\rangle_{i}=$ - $\left|u_{1}\left(A_{i}\right)\right\rangle_{i}$, and $S_{z}^{i}\left|u_{3}\left(A_{i}\right)\right\rangle_{i}=\left|u_{3}\left(A_{i}\right)\right\rangle_{i}$. The ground state is always $\left|u_{0}\left(A_{i}\right)\right\rangle_{i}$. The energy differences $\Delta_{k 0}^{i} \equiv$ $E_{k}^{i}\left(A_{i}\right)-E_{0}^{i}\left(A_{i}\right)$ between the $k$ th excited states and the ground state are estimated as follows: $\Delta_{10}^{i} \simeq 2 A_{i}+$ $2 g_{n} \mu_{n} B, \Delta_{20}^{i} \simeq 2 \epsilon$, and $\Delta_{30}^{i} \simeq 2 \epsilon+2 A_{i}-2 g_{n} \mu_{n} B$. The values of two terms in $\Delta_{10}^{i}$ are of the same order of magnitude as HY; it is possible to control the phase difference between $\left|u_{0}\left(A_{i}\right)\right\rangle_{i}$ and $\left|u_{1}\left(A_{i}\right)\right\rangle_{i}$, varying the magnitude of the HY, $A_{i}(t)$. Note that these two states are robust against this controlling process because they are the eigenstates of temporal Hamiltonian $H^{i}(t)$. Consequently, we choose the quantum computational bases for a single qubit as $\left|u_{0}\left(A_{i}\right)\right\rangle_{i}$ and $\left|u_{1}\left(A_{i}\right)\right\rangle_{i}$. In particular, we write $|0\rangle_{L, i} \equiv\left|u_{0}\left(A_{0}\right)\right\rangle_{i}$ and $|1\rangle_{L, i} \equiv\left|u_{1}\left(A_{0}\right)\right\rangle_{i}$ when $A_{i}(t)=A_{0}$.

Next, we discuss polarization of the electron spins. Taking account of $\sin \theta_{i} \simeq 10^{-3}$ for $A_{i}=A_{0}$, we may regard $\left|u_{0}\left(A_{i}\right)\right\rangle_{i} \simeq|\downarrow\rangle_{i}|0\rangle_{i}$, and the electrons are almost polarized in the downward direction of the $z$ axis. If the system is a thermal equilibrium state with the temperature $T=100 \mathrm{mK}\left(\beta^{-1}=k_{B} T \simeq 8.62 \times 10^{-6} \mathrm{eV}\right)$, which is a typical value in Kane's model [5], the ratio of the number of upper polarized electrons in the $i$ th site, $n_{\uparrow}^{i e}$, to the number of downward polarized ones in it, $n_{\downarrow}^{i e}$, is estimated as follows:

$$
\begin{aligned}
\frac{n_{\uparrow}^{i e}}{n_{\downarrow}^{i e}} & \equiv \frac{\operatorname{Tr}\left(|\uparrow\rangle_{i}\langle\uparrow| e^{-\beta H^{i}}\right)}{\operatorname{Tr}\left(|\downarrow\rangle_{i}\langle\downarrow| e^{-\beta H^{i}}\right)} \\
& =\frac{e^{-\beta \Delta_{30}^{i}}+\cos ^{2} \theta_{i} e^{-\beta \Delta_{20}^{i}}+\sin ^{2} \theta_{i}}{\sin ^{2} \theta_{i} e^{-\beta \Delta_{20}^{i}}+e^{-\beta \Delta_{10}^{i}}+\cos ^{2} \theta_{i}} \\
& \simeq 10^{-5}
\end{aligned}
$$

Here, we have used the following estimation: $\sin \theta_{i} \simeq$ $10^{-3}, 2 \beta \epsilon \simeq 2 \mu_{B} B / k_{B} T \simeq 10$, and $2 \beta A_{0} \simeq 10^{-2}$. The result, $n_{\uparrow}^{i e} / n_{\downarrow}^{i e} \simeq 10^{-5}$, also suggests that the electron spins should be $|\downarrow\rangle_{i}$. However, the above consideration is based on the static physical property; it is nontrivial that it could sustain the downward polarization through the temporal controlling process.

Now, we address the preparation of an initial state. The thermal equilibrium state of the system $\rho_{e q}=$ $\otimes_{i}\left(e^{-\beta H^{i}} / \operatorname{Tr} e^{-\beta H^{i}}\right)$ is not a pure state, even if the temperature $T$ is $100 \mathrm{mK}$. In fact, we find $\rho_{e q}^{i} \simeq$ $0.508\left|u_{0}\left(A_{i}\right)\right\rangle_{i}\left\langle u_{0}\left(A_{i}\right)|+0.492| u_{1}\left(A_{i}\right)\right\rangle_{i}\left\langle u_{1}\left(A_{i}\right)\right|$ for $T=$ $100 \mathrm{mK}, A_{i}=A_{0}, J=0$, and $B=2.0 \mathrm{~T}$. The initialization problem might be solved by the standard scheme in liquid NMR (e.g., by the use of effective pure states). 
Throughout this paper, we investigate only whether a given pure state evolves to a desired state by controlling processes. Such consideration is meaningful, because any arbitrary mixed state is represented by suitable linear combinations of pure states, and the control of a quantum system according to our expectations will be necessary to overcome initialization problems.

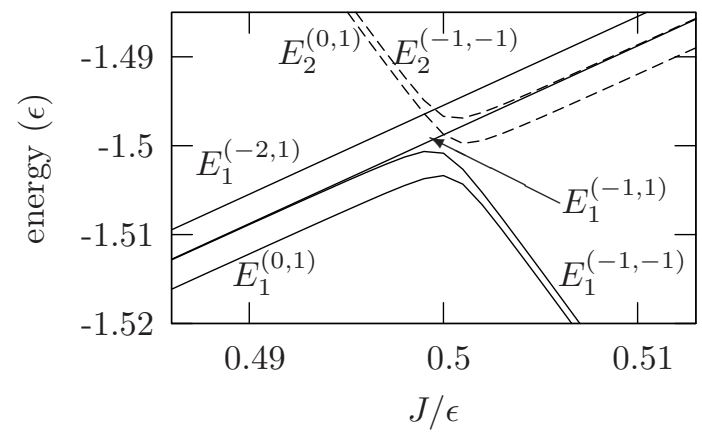

FIG. 1: Configurations of the $H_{C}$ energy level around the level crossing point $\left(J \simeq \mu_{B} B / 2\right)$. Four solid lines represent the lowest eigenvalues of $H_{C}^{(0,1)}, H_{C}^{(-1,-1)}, H_{C}^{(-1,1)}$, and $H_{C}^{(-2,1)}$, respectively, which are associated with the computational bases. Two dashed lines represent the second lowest eigenvalues for each $H_{C}^{(0,1)}$ and $H_{C}^{(-1,1)}$.

Finally, let us consider the eigenvalue problem of the two qubit Hamiltonian

$$
H_{C}(t) \equiv \sum_{i=1}^{2} H^{i}(t)+J(t) \boldsymbol{\sigma}^{1 e} \cdot \boldsymbol{\sigma}^{2 e},
$$

fixing the values of $A_{1}(t), A_{2}(t)$, and $J(t)$. The Hamiltonian $H_{C}(t)$ is composed of the first and the second terms in Eq. (1) and plays a central role in the controlled operations. Hereafter, we write $|\psi\rangle_{i}|\phi\rangle_{i}$ as $|\psi \phi\rangle_{i}$ (e.g., $\left.|\downarrow\rangle_{i}|1\rangle_{i}=|\downarrow 1\rangle_{i}\right)$. We introduce the total spin operator in the direction of the $z$ axis $S_{z}^{\text {tot }}=\left(\sigma_{z}^{1 n}+\sigma_{z}^{1 e}+\right.$ $\left.\sigma_{z}^{2 n}+\sigma_{z}^{2 e}\right) / 2$ and the parity operator, $P$ defined as follows: $P^{\dagger} \sigma_{a}^{1 \alpha} P=\sigma_{a}^{2 \alpha}$ and $P^{\dagger} \sigma_{a}^{2 \alpha} P=\sigma_{a}^{1 \alpha}(\alpha=e, n$ and $a=x, y, z)$. The operator $P$ is an idempotent operator: $P^{2}=\mathbb{1}$. We find that $S_{z}^{\text {tot }}$ always commutes with both $H_{C}(t)$ and $P$, and $P$ commutes with $H_{C}(t)$ if $A_{1}(t)=A_{2}(t)$. As result, in the case of $A_{1}(t)=A_{2}(t)$, the Hamiltonian $H_{C}(t)$ becomes a block diagonal matrix whose blocks are characterized by the eigenvalues of $S_{z}^{\text {tot }}$ and $P, s\left(\in \sigma\left(S_{z}^{\text {tot }}\right) \equiv\{2,1,0,-1,-2\}\right)$ and $p$ $(\in \sigma(P) \equiv\{1,-1\})$, respectively:

$$
H_{C}(t)=\bigoplus_{s, p} H_{C}^{(s, p)}(t)
$$

Note that $S_{z}^{\text {tot }} H_{C}^{(s, p)}(t)=H_{C}^{(s, p)}(t) S_{z}^{\text {tot }}=s H_{C}^{(s, p)}(t)$ and $P H_{C}^{(s, p)}(t)=H_{C}^{(s, p)}(t) P=p H_{C}^{(s, p)}(t)$. A similar result is given by Berman et al. [17], but our computational bases are different from theirs. The submatrices $H_{C}^{(s, p)}(t)$ in Eq. (2) are $4 \times 4$ ones at most.
Hence, we calculate analytically the diagonal form of Eq. (2). We show the explicit forms of its eigenvalues and eigenstates in Appendix $\mathrm{A}$ We explain the relationship between the eigenstates of $H_{C}(t)$ and the computational bases. If the value of $J(t)$ is vanishing, we find that $\left|v_{1}\right\rangle \equiv|0\rangle_{L, 1}|0\rangle_{L, 2},\left|v_{+}\right\rangle \equiv\left(|0\rangle_{L, 1}|1\rangle_{L, 2}+\right.$ $\left.|1\rangle_{L, 1}|0\rangle_{L, 2}\right) / \sqrt{2},\left|v_{-}\right\rangle \equiv\left(|0\rangle_{L, 1}|1\rangle_{L, 2}-|1\rangle_{L, 1}|0\rangle_{L, 2}\right) / \sqrt{2}$, and $\left|v_{4}\right\rangle \equiv|1\rangle_{L, 1}|1\rangle_{L, 2}$ are the eigenstates of $H_{C}^{(0,1)}(t)$, $H_{C}^{(-1,1)}(t), H_{C}^{(-1,-1)}(t)$, and $H_{C}^{(-2,+1)}(t)$, respectively. Therefore, these four states play an essential role in the scheme of controlled operations. In Fig. 1 we show that the eigenvalues of submatrices related to the computational bases around the level crossing $\left(J \simeq \mu_{B} B / 2\right)$, varying the magnitude of the $\mathrm{EE}, J$. The eigenvalues of $H_{C}^{(s, p)}$ are specified by $E_{k}^{(s, p)}(k=1,2$ for $(s, p)=$ $(0,1),(-1,1)$, and $k=1$ for $(s, p)=(-1,1),(-2,1))$. The energy difference $E_{1}^{(-1,1)}-E_{1}^{(-1,-1)}$ is tiny and positive for $J<\mu_{B} B / 2$. As $J$ increases, the levels $E_{1}^{(0,1)}$ and $E_{1}^{(-1,-1)}$ approach $E_{2}^{(0,1)}$ and $E_{2}^{(-1,-1)}$, respectively.

\section{THE QUANTUM GATES}

\section{A. The phase-shift operations}

We achieve the phase-shift operation for the $i$ th qubit, varying $A_{i}(t)$ adiabatically with the other parameters fixed; $J(t)=0, B_{a c}=0$, and $A_{j}(t)=A_{0}(j \neq i)$.

First, we calculate the time-evolution operator for the $i$ th qubit:

$$
U^{i}\left(t_{Z}\right)=T\left\{\exp \left[-\frac{i}{\hbar} \int_{0}^{t_{Z}} H^{i}(t) d t\right]\right\} .
$$

The symbol $T$ means the time-ordered product. We define the operation time for the phase-shift operation as $t_{Z}(>0)$. Introducing a dimensionless parameter $a$ $(0<a<1)$ and a dimensionless variable $\tau=t / t_{Z}$, we assume the time dependence of $A_{i}(t)$ as follows (see, Fig.(2):

$$
A_{i}(t)=\left\{\begin{array}{ll}
A_{0} f_{A}(\tau) & \left(0 \leq \tau \leq \frac{1}{2}\right) \\
A_{0} f_{A}(1-\tau) & \left(\frac{1}{2} \leq \tau \leq 1\right)
\end{array} .\right.
$$

The function $f(\tau)$ in Eq. (4) is defined as

$$
\begin{aligned}
f_{A}(\tau)=\Theta( & \left.\frac{1}{4}-\tau\right)\left(1-8 a \tau^{2}\right) \\
& +\Theta\left(\tau-\frac{1}{4}\right)\left[1-a+8 a\left(\tau-\frac{1}{2}\right)^{2}\right],
\end{aligned}
$$

and $\Theta(\tau)$ is the Heaviside function. The initial state for the $i$ th qubit will be spanned by $|0\rangle_{L, i}$ and $|1\rangle_{L, i}$, because $A_{i}(0)=A_{0}$. We also find that $\left|u_{0}\left(A_{i}\left(t_{Z}\right)\right)\right\rangle_{i}=|0\rangle_{L, i}$ and $\left|u_{1}\left(A_{i}\left(t_{Z}\right)\right)\right\rangle_{i}=|1\rangle_{L, i}$, because we assume that the time dependence of $A_{i}(t)$ is given by Eq. (44). Consequently, 


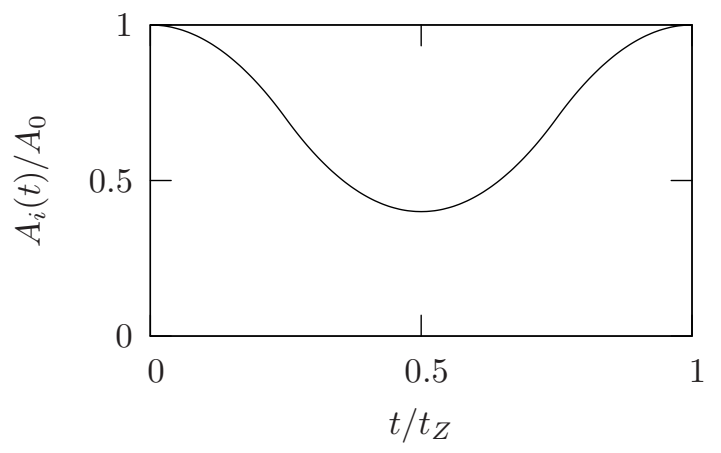

FIG. 2: Profile of $A_{i}(t)(a=0.6)$.

Eq. (3), due to the adiabatic theorem [18, 19], is written by

$$
U^{i}\left(t_{Z}\right)=e^{-i \delta_{0}^{i}}|0\rangle_{L, i}\left\langle 0\left|+e^{-i \delta_{1}^{i}}\right| 1\right\rangle_{L, i}\langle 1|+R^{i},
$$

where $\delta_{0}^{i}=t_{Z} h^{-1} \int_{0}^{1}\left[-A_{i}(s)-\sqrt{\epsilon^{2}+4 A_{i}(s)^{2}}\right] d s$ and $\delta_{1}^{i}=t_{Z} \hbar^{-1}\left[g_{n} \mu_{n} B-\mu_{B} B+\int_{0}^{1} A_{i}(s) d s\right]$. The third term in Eq. (15), $R^{i}$ includes $\left|u_{2}\left(A_{i}\left(t_{Z}\right)\right)\right\rangle_{i}\left\langle u_{0}\left(A_{i}\left(t_{Z}\right)\right)\right|$ and $\left|u_{0}\left(A_{i}\left(t_{Z}\right)\right)\right\rangle_{i}\left\langle u_{2}\left(A_{i}\left(t_{Z}\right)\right)\right|$ mediated by the level crossing effect in the course of operation. By the definition of a matrix norm [20] for an $n \times n$ complex matrix $M=\left(M_{a b}\right),\|M\| \equiv \max _{1 \leq a, b \leq n}\left|M_{a b}\right|,\left\|R^{i}\right\|$ is estimated as the order of $\hbar / 2 \epsilon t_{Z}$, and later on we find that it's very small. A criterion for the validity of the adiabatic ap- proximation is given as follows [19]:

$$
\max _{0 \leq \tau \leq 1}\left|\frac{\left\langle u_{2}^{i}(\tau)\left|d H^{i}(\tau) / d \tau\right| u_{0}^{i}(\tau)\right\rangle}{E_{2}^{i}(\tau)-E_{0}^{i}(\tau)}\right|<1 .
$$

In this case, we have a condition,

$$
\frac{\epsilon A_{0}}{\epsilon^{2}+4 A_{0}^{2}} a<1
$$

The inequality (6) is always fulfilled, because $O\left(2 A_{0} / \epsilon\right) \sim$ $10^{-3}$ and $O(a) \sim 1$.

Next, we calculate the time-evolution operator for the remaining $j$ th qubit $(j \neq i)$. Taking account of the constancy of $A_{j}(t)=A_{0}$ and the initial states for the $j$ th qubit spanned by $|0\rangle_{L, j}$ and $|1\rangle_{L, j}$, we simply obtain the following result:

$$
U^{j}\left(t_{Z}\right)=e^{-i \delta_{0}^{j}}|0\rangle_{L, j}\left\langle 0\left|+e^{-i \delta_{1}^{j}}\right| 1\right\rangle_{L, j}\langle 1|,
$$

where $\delta_{0}^{j}=t_{Z} \hbar^{-1}\left(-A_{0}-\sqrt{\epsilon^{2}+4 A_{0}^{2}}\right)$ and $\delta_{1}^{j}=$ $t_{Z} \hbar^{-1}\left(g_{n} \mu_{n} B-\mu_{B} B+A_{0}\right)$.

Now, we construct the phase-shift operation (i.e., a $Z$ rotation) with the given angle $\theta_{Z}$ for the $i$ th qubit. In the first place, the phase difference between $|0\rangle_{L, i}$ and $|1\rangle_{L, i}$ should be equal to $\theta_{Z} ; \delta_{0}^{i}-\delta_{1}^{i}=\theta_{Z}+2 n \pi(n \in \mathbb{Z})$, due to Eq. (5). Secondly, for all qubits except for the $i$ th qubit, no phase differences have to exist; $\delta_{0}^{j}-\delta_{1}^{j}=2 m \pi$ $(m \in \mathbb{Z}, m \neq 0$, and $j \neq i)$, due to Eq. (17). Using these two conditions, we obtain the following equations:

$$
\begin{aligned}
& \frac{\epsilon-2 g_{n} \mu_{n} B+\int_{0}^{1}\left[-2 A_{i}(s)-\sqrt{\epsilon^{2}+4 A_{i}(s)^{2}} d s\right]}{\epsilon-2 g_{n} \mu_{n} B-2 A_{0}-\sqrt{\epsilon^{2}+4 A_{0}^{2}}}=\frac{\theta_{Z}+2 n \pi}{2 m \pi}, \\
& t_{Z}=\frac{2 m \pi \hbar}{\epsilon-2 g_{n} \mu_{n} B-2 A_{0}-\sqrt{\epsilon^{2}+4 A_{0}^{2}}} .
\end{aligned}
$$

We find that Eq. (8) includes only one unknown parameter $a$, except for the free parameters $m$ and $n$. We should choose suitable values for them so that the solution of $O(a) \sim 1$ exists for any $\theta_{Z}$; actually, we choose $m=-5$ and $n=-6$. We numerically solve Eq. (8) by the bisection method. Then, we get the operation time $t_{Z}$ by substituting the value of $m$ for Eq. (9); its value is independent of the value of $\theta_{Z}$.

We show the results in Table The minimum value of $A_{i}(t)$ is given by $A_{0}(1-a)$. The phase-shift operations of the angle $\theta_{Z}=\pi / 4$ (i.e., the $\pi / 8$ gate) and $\pi / 2$ (i.e., the phase gate) are performed by reducing the HY by up to about $66 \%$ during the operation time, which is about two times longer than that of Ref. [15]. This difference comes from our chosen profile function of $A_{i}(t)$, valid for the adiabatic approximation. The value of $\hbar / 2 \epsilon t_{Z}$ is approx-
TABLE I: Calculated values of $a$ and $t_{Z}$ with $m=-5$ and $n=-6$.

\begin{tabular}{lcc}
\hline \hline$\theta_{Z}$ & $a$ & $t_{Z}(\mu s)$ \\
\hline$\pi / 4$ & 0.598 & 0.05 \\
$\pi / 2$ & 0.664 & 0.05 \\
\hline \hline
\end{tabular}

imately estimated as $A_{0} / 4|m| \pi \epsilon ; A_{0} / 4|m| \pi \epsilon \simeq 10^{-5}$ for $m=-5$; the correction to the adiabatic approximation is very small. The choice of $m$ and $n$ is not unique. In fact, we find that other solutions of Eq. (8) exist if $m \leq-5$ and $n \leq-6$. The operation time $t_{Z}$ for $(m, n)=(-5,-6)$ is the shortest of the solutions. We confirm also the realization of the desired phase-shift gates by solving numerically the time-dependent Schrödinger equation for 
the resultant parameters.

We summarize the results for the phase-shift operation. We have achieved it by adiabatically controlling the magnitude of the $\mathrm{HY}, A_{i}(t)$, with $J(t)=0, A_{j}(t)=A_{0}$ $(j \neq i)$, and $B_{a c}=0$. Its errors are very small, as the adiabatic approximation works well. The phase-shift operations relevant to universal quantum gates, the $\pi / 8$ gate $\left(\theta_{Z}=\pi / 4\right)$ and the phase gate $\left(\theta_{Z}=\pi / 2\right)$, are realized by reducing the value of $A_{i}(t)$ from $A_{0}$ to about $0.4 A_{0}$ at most during the operation time $t_{Z}(\simeq 0.05 \mu \mathrm{s})$. According to [5], it might be possible to decrease the magnitude of the HY by up to $50 \%$. This means that further development of the experimental techniques might be needed to fully realize them by our scheme.

\section{B. The spin-flip operations}

Let us consider the scheme of the spin-flip operation. We reproduce the spin-flip operation in Ref. [15] by applying the transverse time-dependent magnetic field (see the third term in Eq. (10), using an approximation. The idea is based on a standard technique in NMR 21, 22]. Here, we assume that the transverse magnetic field is turned on or off instantaneously. We show that a serious error exists in Ref. 15], even if such an ideal controlling process is realized. Hereafter, we concentrate on the scheme of an $\mathrm{X}$ rotation for the $i$ th qubit.

First of all, we explain the controlling processes of the $\mathrm{EE}$, the $\mathrm{HY}$, and the transverse time-dependent magnetic field. We keep $J(t)=0$ and $A_{j}(t)=A_{0}(j \neq i)$. By contrast, we adiabatically change the magnitude of $A_{i}(t)$; in the first place, we decrease the value of $A_{i}(t)$ up to a suitable constant value $A\left(<A_{0}\right)$ (we explain how to choose the value of $A$ below) during the time interval $t_{X}^{\prime} / 2$ (the first step), keep $A_{i}(t)=A$ during the time interval $t_{X}$ (the second step), and finally increase it from $A$ to $A_{0}$ (the third step). Thus, the time dependence of $A_{i}(t)$ is given by

$$
A_{i}(t)=\left\{\begin{array}{ll}
A_{0} f_{A}(\tau) & \left(0 \leq \tau \leq \frac{1}{2}\right) \\
A & \left(\frac{1}{2} \leq \tau \leq \tau_{s p}-\frac{1}{2}\right) \\
A_{0} f_{A}\left(\tau_{s p}-\tau\right) & \left(\tau_{s p}-\frac{1}{2} \leq \tau \leq \tau_{s p}\right)
\end{array},\right.
$$

where $\tau=t / t_{X}^{\prime}, \tau_{X}=t_{X} / t_{X}^{\prime}$, and $\tau_{s p}=1+\tau_{X}$. The parameter $a$ in $f_{A}(\tau)$ is given by $a=1-A / A_{0}$. In addition, the transverse time-dependent magnetic field is globally applied, while we keep $A_{i}(t)=A$ (the second step).

A undesired phase difference between $|0\rangle_{i}$ and $|1\rangle_{i}$ is caused by the adiabatically varying processes during $A_{i}(t)$ (the first and third steps). This phase difference can be canceled by carrying out a suitable $\mathrm{Z}$ rotation, as described in Sec.IIIA before and after the first and third steps respectively.

Let us discuss the Hamiltonian in the second step. After instantaneously turning on the transverse magnetic field, the Schrödinger equation for the $i$ th qubit is written from Eq. (11):

$$
i \hbar \frac{d}{d t}|\psi(t)\rangle_{i}=\left[H^{i}+H_{a c}^{i}(t)\right]|\psi(t)\rangle_{i} .
$$

We solve Eq. (11) in the rotating frame 21, 22]; we remove the time dependence of the Hamiltonian in Eq. (11) by applying the unitary operator $D_{z}\left(t ; \omega_{a c}\right) \equiv$ $\exp \left[-i \omega_{a c} t\left(\sigma_{z}^{i e}+\sigma_{z}^{i n}\right) / 2\right] . \quad$ Introducing $\left|\psi_{\text {rot }}(t)\right\rangle_{i} \equiv$ $D_{z}\left(t ; \omega_{a c}\right)|\psi(t)\rangle_{i}$, we obtain the following Schrödinger equation in the rotating frame from Eq. (11):

$$
i \hbar \frac{d}{d t}\left|\psi_{\text {rot }}(t)\right\rangle_{i}=H_{\text {rot }}^{i}\left|\psi_{\text {rot }}(t)\right\rangle_{i}
$$

where $H_{r o t}^{i}=\hbar \omega^{e} \sigma_{z}^{i e} / 2-\hbar \omega^{n} \sigma_{z}^{i n} / 2+A \boldsymbol{\sigma}^{i e} \cdot \boldsymbol{\sigma}^{i n}+$ $\mu_{B} B_{a c} \sigma_{x}^{i e}-g_{n} \mu_{n} B_{a c} \sigma_{x}^{i n}, \hbar \omega^{e}=2 \mu_{B} B+\hbar \omega_{a c}$, and $\hbar \omega^{n}=2 g_{n} \mu_{n} B-\hbar \omega_{a c}$. We naturally expand $\left|\psi_{\text {rot }}(t)\right\rangle_{i}$ and $H_{\text {rot }}^{i}$ by $\left\{\left|u_{k}(A)\right\rangle_{i}\right\}$. Let us introduce the matrix representation for an operator $M$ as follows: $M_{a b} \equiv$ ${ }_{i}\left\langle u_{a}(A)|M| u_{b}(A)\right\rangle_{i}(a, b=0,1,2,3)$. Thus, the Hamiltonian in the rotating frame, $H_{r o t}^{i}$, is written down as the sum of a block diagonal matrix $H_{d}^{i}$ and a off-diagonal matrix $H_{m i x}^{i}$ :

$$
\begin{aligned}
H_{\text {rot }}^{i} & =H_{d}^{i}+H_{m i x} \\
& =\left(\begin{array}{cccc}
E_{0}^{i}(A) & -\nu_{\theta} B_{a c} & 0 & 0 \\
-\nu_{\theta} B_{a c} & E_{1}^{i}(A)-\hbar \omega_{a c} & 0 & 0 \\
0 & 0 & E_{2}^{i}(A) & -\nu_{-\theta} B_{a c} \\
0 & 0 & -\nu_{-\theta} B_{a c} & E_{3}^{i}(A)+\hbar \omega_{a c}
\end{array}\right)+\left(\begin{array}{cccc}
0 & 0 & 0 & \mu_{-\theta} B_{a c} \\
0 & 0 & \mu_{\theta} B_{a c} & 0 \\
0 & \mu_{\theta} B_{a c} & 0 & 0 \\
\mu_{-\theta} B_{a c} & 0 & 0 & 0
\end{array}\right) .
\end{aligned}
$$

Here, we define $\mu_{\theta}=\mu_{B} \cos \theta-g_{n} \mu_{n} \sin \theta$ and $\nu_{\theta}=$ $\mu_{B} \sin \theta+g_{n} \mu_{n} \cos \theta$, and the mixing angle $\theta$ is defined for $\theta_{i}$ when $A_{i}(t)=A$. Whenever one can safely assume that the off-diagonal Hamiltonian, $H_{m i x}^{i}$, is negligible, the computational bases, $\left|u_{0}(A)\right\rangle_{i}$ and $\left|u_{1}(A)\right\rangle_{i}$, do not mix with the remaining irrelevant states, $\left|u_{2}(A)\right\rangle_{i}$ and $\left|u_{3}(A)\right\rangle_{i}$, because of the block diagonal Hamiltonian, $H_{d}^{i}$. In most studies up to now, this approximation has been 
taken for granted.

However, it is nontrivial whether we should omit $H_{m i x}^{i}$ in solving Eq. (12). Notice that $\mu_{\theta}$ is larger than $\nu_{\theta}$, unless $\theta$ is close to $\pi / 2$. We show that a considerable error is caused by neglecting $H_{m i x}^{i}$ below.

Before discussing such an error, we reproduce the results in Ref. 15. by means of our computational bases. We solve Eq. (12), omitting $H_{m i x}^{i}$. We choose the value of $A$ so as to satisfy the Larmor resonance condition corresponding to the computational bases,

$$
\hbar \omega_{a c}=E_{1}^{i}(A)-E_{0}^{i}(A) .
$$

Taking account of Eq. (14) and the initial state $|\phi\rangle_{i}$ expanded by $\left|u_{0}(A)\right\rangle_{i}$ and $\left|u_{1}(A)\right\rangle_{i}$ (i.e., the state just before turning on the transverse magnetic field), we obtain the resultant state at $t_{X}$ in the rotating frame:

$$
\left|\psi_{\text {rot }}\left(t_{X}\right)\right\rangle_{i}=e^{-\frac{i}{\hbar} t_{X} E_{0}^{i}(A)} e^{i \theta_{X} \sigma_{X}^{i}(A)}|\phi\rangle_{i} .
$$

We define $\sigma_{X}^{i}(A)=\left|u_{0}(A)\right\rangle_{i}\left\langle u_{1}(A)|+| u_{1}(A)\right\rangle_{i}\left\langle u_{0}(A)\right|$ and $\theta_{X}=\nu_{\theta} B_{a c} t_{X} / \hbar$. Accordingly,

$$
\left|\psi\left(t_{X}\right)\right\rangle_{i}=e^{-\frac{i}{\hbar} t_{X} E_{0}^{i}(A)} D_{z}^{\dagger}\left(\omega_{a c} t_{X}\right) e^{i \theta_{X} \sigma_{X}^{i}(A)}|\phi\rangle_{i} .
$$

Under the Larmor resonance condition (14) for the $i$ th qubit, the time-evolution of the remaining $j$ th qubit $(j \neq i)$ is approximately expressed by a $\mathrm{Z}$ rotation. Moreover, both the overall phase and $D_{z}^{\dagger}\left(\omega_{a c} t_{X}\right)$ in Eq. (16) are canceled by carrying out suitable $\mathrm{Z}$ rotations. Consequently, we make only the state of the target qubit flip due to Eq. (16). In this way, we have reproduced a result corresponding to that achieved in Ref. [15].

Now, we demonstrate the existence of a considerable error in the above scheme. We solve Eq. (12) without omitting $H_{m i x}^{i}$. After this calculation, we compare the exact solution with the approximate solution (15) by calculating the following quantity:

$$
F\left(|\phi\rangle_{i}\right)=\left|{ }_{i}\left\langle\phi\left|e^{\frac{i}{\hbar} H_{r o t}^{i} t_{X}} e^{-\frac{i}{\hbar} H_{d}^{i} t_{X}}\right| \phi\right\rangle_{i}\right| .
$$

This is just the fidelity [16] between the aimed state $e^{-\frac{i}{\hbar} H_{d}^{i} t_{X}}|\phi\rangle_{i}$ obtained by an X rotation and the physical state $e^{-\frac{i}{\hbar} H_{\text {rot }}^{i} t_{X}}|\phi\rangle_{i}$ obtained by Eq. (12). If the value of $F\left(|\phi\rangle_{i}\right)$ is adequately close to one, the dynamics generated only by $H_{d}^{i}$ is a good approximation for the genuine one; we can achieve an $\mathrm{X}$ rotation by Hill and Goan's scheme. The exact solution can be represented by the eigenvalues and the eigenvectors of Eq. (13), which are given in Appendix $\mathbb{B}$ Adopting the typical value of the transverse magnetic field, $B_{a c}=2.5 \mathrm{mT}$, and choosing $\theta_{X}=\pi / 4$, we calculate $F\left(|\phi\rangle_{i}\right)$ for three values of the $\mathrm{HY}: A / A_{0}=0.75,0.5,0.25$, and different two initial states. The $\mathrm{X}$ rotation for $\theta_{X}=\pi / 4$ plays an essential role in the Hadamard gate $U_{H}: U_{H}=$ $-i e^{i \pi \sigma_{z} / 4} e^{i \pi \sigma_{x} / 4} e^{i \pi \sigma_{z} / 4}$. We show the results in Table III They imply that the values of $F\left(|\phi\rangle_{i}\right)$ are almost on the order of $10^{-1}$, regardless of the initial states and the magnitude of the $\mathrm{HY}$; the $\mathrm{X}$ rotation given
TABLE II: Calculated values of the fidelity (17) with $\theta_{X}=$ $\pi / 4$ and $B_{a c}=2.5 \mathrm{mT}$.

\begin{tabular}{ccc}
\hline \hline & \multicolumn{2}{c}{$F\left(|\phi\rangle_{i}\right)$} \\
$A / A_{0}$ & $|\phi\rangle_{i}=|0\rangle_{i}$ & $|\phi\rangle_{i}=\left(|0\rangle_{i}+|1\rangle_{i}\right) / \sqrt{2}$ \\
\hline 0.75 & 0.72514 & 0.70458 \\
0.5 & 0.72458 & 0.70454 \\
0.25 & 0.73390 & 0.70526 \\
\hline \hline
\end{tabular}

by Eq. (16) involves a serious error, whose probability, $1-\max _{|\phi\rangle_{i}}\left[F\left(|\phi\rangle_{i}\right)\right]$, is estimated at about $10^{-1}$. According to Ref. [15], the error probability in terms of the fidelity (i.e., $1-\max _{|\phi\rangle_{i}}\left[F\left(|\phi\rangle_{i}\right)\right]$ ) is estimated about at $10^{-5}$ in the CNOT gate. We will need to discuss the possibility of quantum error correction 23]. However, it is certain that the error in the X rotation in Ref. [15] is vastly larger than the one estimated there.

The presence of the error means that $H_{m i x}^{i}$ in Eq. (13) shouldn't be regarded as a perturbation; the bases where $H_{d}^{i}$ is a diagonal matrix are not suitable for the analysis of the Schrödinger equation (12). The above consideration is self-evident, investigating the time evolution operator under the Lamour resonance condition (14). The dynamics by $H_{d}^{i}$, which is relevant to the $\mathrm{X}$ rotation, is characterized by the energy scale $\nu_{\theta} B_{a c}$. However, we usually find $\nu_{\theta}<\mu_{\theta}$. Consequently, the mixing effect by $H_{m i x}^{i}$ becomes dominant. We also recognize the dominant effect of $H_{m i x}^{i}$ from the following viewpoint of estimating errors. If we simply apply the ordinal perturbation method to the calculation of the eigenvectors of $H_{\text {rot }}^{i}$ in Eq. (17), we obtain the following result for $|\phi\rangle_{i}=|0\rangle_{i}$ under the condition (14):

$$
F\left(|0\rangle_{i}\right)=\sqrt{\frac{1}{2}+\frac{1}{2} \cos \left[\frac{t_{X}}{\hbar}\left(\tilde{\Delta}_{1}^{i}-\tilde{\Delta}_{0}^{i}\right)\right]}+O\left(\frac{B_{a c}^{2}}{B^{2}}\right) .
$$

Here, we regard $H_{d}^{i}$ as the free part in (13). We define the difference between the lowest eigenvalue of $H_{d}^{i}$ and the one of $H_{\text {rot }}^{i}$ as $\tilde{\Delta}_{0}^{i}$. We also define the difference between the second lowest eigenvalue of $H_{d}^{i}$ and the one of $H_{\text {rot }}^{i}$ as $\tilde{\Delta}_{1}^{i}$. Indeed, we find $t_{X}\left(\tilde{\Delta}_{1}^{i}-\tilde{\Delta}_{0}^{i}\right) / \hbar \approx 10^{-6}$ for $A / A_{0}=0.5$ and $\theta_{X}=\pi / 4$. Then, we approximately get $F\left(|0\rangle_{i}\right) \approx 1$ for $A / A_{0}=0.5$ and $\theta_{X}=\pi / 4$; this result is clearly different from the rigorous one.

In summary, we have shown that the $\mathrm{X}$ rotation in Ref. [15] includes a considerable error, which is generated by $H_{m i x}^{i}$. The mixing Hamiltonian $H_{m i x}^{i}$ never appears under the assumption that the electron spin state is always down, and this term should not be regarded as a perturbation for $H_{d}^{i}$.

\section{The controlled $-\mathrm{Z}$ gates}

In Sec.IIIB we explained that there is considerable error in the $\mathrm{X}$ rotation carried out according to Hill and 
Goan's scheme. Generally, spin-flip operations play an important role in controlled operations. In Ref. 15], they are frequently used even in the controlled $-\mathrm{Z}$ gates. Here, we show that the error in the controlled $-\mathrm{Z}$ gate mainly occurs in the spin-flip operations.

First, by means of the adiabatic theorem, we calculate the time evolution operator for two qubits during an interval $t_{C}$,

$$
U_{C}\left(t_{C}\right)=T\left[\exp \left(-\frac{i}{\hbar} \int_{0}^{t_{C}} H_{C}(t) d t\right)\right],
$$

keeping $A_{1}(t)=A_{2}(t)=A_{0}$, turning off the transverse magnetic field, and adiabatically varying $J(t)$. The total operation time of $J(t)$ is defined as $t_{C}$. In order to get an analytical expression of the operator, we take the following profile of $J(t)$, introducing four parameters: The maximum value of $J(t), J_{c}$, the period $t_{h}$ when $J(t)$ keeps $J_{c}$, the former and the latter periods when $J(t)$ varies adiabatically, $t_{a}$ (i.e., $t_{C}=t_{a}+t_{h}$ ), and the smoothness of profile touching to the constant line, $\tau^{\prime}$. The assumed time dependence of $J(t)$ is as follows:

$$
J(t)=\left\{\begin{array}{ll}
J_{c} f_{J}(\tau) & \left(0 \leq \tau \leq \frac{1}{2}\right) \\
J_{c} & \left(\frac{1}{2} \leq \tau \leq \tau_{C}-\frac{1}{2}\right) \\
J_{c} f_{J}\left(\tau_{C}-\tau\right) & \left(\tau_{C}-\frac{1}{2} \leq \tau \leq \tau_{C}\right)
\end{array},\right.
$$

where $\tau$ is a scaled time $\tau=t / t_{a}, \tau_{C}=t_{C} / t_{a}$, and $\tau_{h}=t_{h} / t_{a}$ (see Fig.(3). The function $f_{J}(\tau)$ in Eq. (19) is defined as,

$$
f_{J}(\tau)=\Theta\left(\tau^{\prime}-\tau\right) \frac{2}{\tau^{\prime}} \tau^{2}+\Theta\left(\tau-\tau^{\prime}\right)\left[1-\frac{(1-2 \tau)^{2}}{1-2 \tau^{\prime}}\right] .
$$

The initial state and final state for two qubits will be spanned by $\left|v_{1}\right\rangle,\left|v_{+}\right\rangle,\left|v_{-}\right\rangle$, and $\left|v_{4}\right\rangle$, because $J(0)=$ $J\left(t_{C}\right)=0$ and $A_{1}(t)=A_{2}(t)=0\left(0 \leq t \leq t_{C}\right)$. Then, the time-evolution operator (18) is given by

$$
U_{C}\left(t_{C}\right)=\sum_{k=1,+,-, 4} e^{-i\left(\alpha_{k}+\beta_{k}\right)}\left|v_{k}\right\rangle\left\langle v_{k}\right|+R_{C} .
$$

The phase $\alpha_{k}$ and $\beta_{k}$ in Eq. (20) are related to the processes in which $J(t)$ varies (i.e., $0 \leq \tau \leq 1 / 2$ and $\tau_{C}-1 / 2 \leq \tau \leq \tau_{C}$ ) and in which $J(t)=J_{c}$ (i.e., $\left.1 / 2 \leq \tau \leq \tau_{C}-1 / 2\right)$, respectively. The analytical expressions for them are shown in Appendix $\$$ The last term in Eq. (201), like the third term in Eq. (5), represents the deviation from the adiabatic approximation and mixes the states relevant to quantum computation with the irrelevant states. This gives a dominant contribution near the level crossing region around $J_{c}=\mu_{B} B / 2$ (see, Fig.11), where we have to apply carefully the adiabatic approximation. In this paper, we investigate Eq. (18) in the regime where the level crossing is not included; we can safely neglect $R_{C}$ in Eq. (20). Actually, we confirm that the adiabatic approximation works very well, solving numerically the time-dependent Schrödinger equation.

Secondly, we explain how to connect $U_{C}\left(t_{C}\right)$ with a controlled- $Z$ gate. Hereafter, we assume that the spinflip operations are realized by using Eq. (16). First of

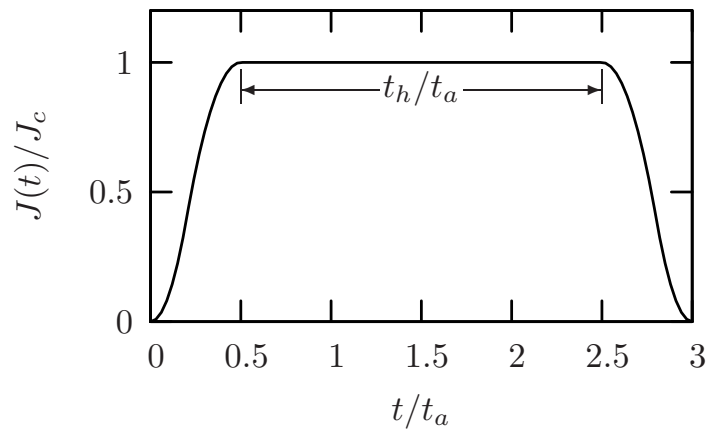

FIG. 3: Profile of $J(t)\left(\tau_{h}=t_{h} / t_{a}=2.0\right.$ and $\left.\tau^{\prime}=0.2\right)$.

all, we rewrite Eq. 201 as $U_{C}\left(t_{C}\right)=U_{a d} U_{s t}$, where $U_{a d}=\sum_{k} e^{-i \alpha_{k}}\left|v_{k}\right\rangle\left\langle v_{k}\right|$ and $U_{s t}=\sum_{k} e^{-i \beta_{k}}\left|v_{k}\right\rangle\left\langle v_{k}\right|$. Next, we investigate $U_{s t}$ in more detail. Introducing $\mathbb{1}_{i} \equiv|0\rangle_{L, i}\langle 0|+| 1\rangle_{L, i}\left\langle 1\left|, \sigma_{i z} \equiv\right| 0\right\rangle_{L, i}\langle 0|-| 1\rangle_{L, i}\langle 1|, \sigma_{i x} \equiv$ $|0\rangle_{L, i}\langle 1|+| 1\rangle_{L, i}\langle 0|$, and $\sigma_{i y} \equiv-i\left(|0\rangle_{L, i}\langle 1|-| 1\rangle_{L, i}\langle 0|\right)$, we find that $U_{s t}$ is given by

$$
U_{s t}=V e^{-i \delta_{C}\left(\sigma_{1 x} \otimes \sigma_{2 x}+\sigma_{1 y} \otimes \sigma_{2 y}\right)} e^{-i \delta_{C}^{\prime} \sigma_{1 z} \otimes \sigma_{2 z}},
$$

where $V=e^{-i c} e^{-i \delta_{s}\left(\sigma_{1 z}+\sigma_{2 z}\right)}, c=\left(\beta_{1}+\beta_{+}+\beta_{-}+\beta_{4}\right) / 4$, $\delta_{s}=\left(\beta_{1}-\beta_{4}\right) / 4, \delta_{C}=\left(\beta_{+}-\beta_{-}\right) / 4$, and $\delta_{C}^{\prime}=\left(\beta_{1}-\beta_{+}-\right.$ $\left.\beta_{-}+\beta_{4}\right) / 4$. Note that this operator contains spin-flip operations. Here, we define the unitary operator $W$ as

$$
\begin{aligned}
W & =\left(U_{H} \otimes U_{H}\right) V^{\dagger} U_{s t}\left(U_{H} \otimes U_{H}\right) \\
& =e^{-i \delta_{C}\left(\sigma_{1 z} \otimes \sigma_{2 z}+\sigma_{1 y} \otimes \sigma_{2 y}\right)} e^{-i \delta_{C}^{\prime} \sigma_{1 x} \otimes \sigma_{2 x}},
\end{aligned}
$$

where $U_{H}$ is the Hadamard gate. Then, according to the idea shown in Ref. [15], we find that the controlled $-Z$ gate $U_{c z}\left(\theta_{c z}\right)$ of the angle $\theta_{c z}$ is achieved as follows:

$$
\begin{aligned}
U_{c z}\left(\theta_{c z}\right)= & e^{-i \frac{\theta_{c z}}{2} \sigma_{1 z} \otimes \sigma_{2} z}\left(\mathbb{1}_{1} \otimes e^{i \frac{\theta_{c z}}{2} \sigma_{2} z}\right) \\
=W\left(\mathbb{1}_{1} \otimes \sigma_{2 z}\right) W & \left(\mathbb{1}_{1} \otimes \sigma_{2 z}\right) \\
& \times\left(\mathbb{1}_{1} \otimes e^{i \frac{\theta_{c z}}{2} \sigma_{2} z}\right) .
\end{aligned}
$$

Note that the several basic identities $\sigma_{i z} \sigma_{i x} \sigma_{i z}=-\sigma_{i x}$, $\sigma_{i z} \sigma_{i y} \sigma_{i z}=-\sigma_{i y}$, and $\left[\sigma_{1 a} \otimes \sigma_{2 a}, \sigma_{1 b} \otimes \sigma_{2 b}\right]=0(a, b=$ $x, y, z)$ play an essential role in the derivation of Eq. (21). The angle $\theta_{c z}$ is given by $\theta_{c z}=4 \delta_{C}=\beta_{+}-\beta_{-}$, from which $t_{h}$ is given by,

$$
t_{h}=\frac{\theta_{c z} \hbar}{2 J_{c}+\sqrt{\left(\epsilon-2 J_{c}\right)^{2}+4 A_{0}^{2}}-\sqrt{\epsilon^{2}+4 A_{0}^{2}}} .
$$

Here, let us recall that the physical time-evolution is given by $U_{C}\left(t_{C}\right)$. The unitary operator $W$ in Eq. (21) is rewritten in the term of $U_{C}\left(t_{C}\right)$ as follows:

$$
W=\left(U_{H} \otimes U_{H}\right) V^{\dagger} U_{a d}^{\dagger} U_{C}\left(t_{C}\right)\left(U_{H} \otimes U_{H}\right) .
$$

The unitary operator $V$ is composed of several $\mathrm{Z}$ rotations.

Now that we find the connection between $U_{C}\left(t_{C}\right)$ and the controlled $-Z$ gate, we should discuss the effect of $U_{a d}$. 
Generally, the unitary operator $U_{a d}$ should include two qubit operations. We would like to represent $U_{a d}$ as single qubit operations, in particular, $\mathrm{Z}$ rotations, because they have been constructed in Sec.IIIA and their errors are very small. We find that $U_{a d}$ is equal to the single qubit operation, $\sigma_{1 z} \otimes \sigma_{2 z}$, if the following conditions are fulfilled: $\alpha_{+}-\alpha_{1}=\pi+2 m_{+} \pi, \alpha_{-}-\alpha_{1}=\pi+2 m_{-} \pi$, and $\alpha_{4}-\alpha_{1}=2 m_{4} \pi\left(m_{+}, m_{-}, m_{4} \in \mathbb{Z}\right.$ and $\left.m_{4} \neq 0\right)$. These three conditions are written by

$$
\begin{aligned}
& \frac{-\sqrt{\epsilon^{2}+4 A_{0}^{2}}-\epsilon+2 g_{n} \mu_{n} B+2 \int_{0}^{\frac{1}{2}}\left[J(\tau)-E_{1}^{(0,+)}(\tau)\right] d \tau}{-2 \epsilon+4 g_{n} \mu_{n} B+2 A_{0}+2 \int_{0}^{\frac{1}{2}}\left[J(\tau)-E_{1}^{(0,+)}(\tau)\right] d \tau}=\frac{1+2 m_{+}}{2 m_{4}}, \\
& \frac{-\epsilon+2 g_{n} \mu_{n} B-2 \int_{0}^{\frac{1}{2}}\left\{\sqrt{[\epsilon-2 J(\tau)]^{2}+4 A_{0}^{2}}+J(\tau)+E_{1}^{(0,+)}(\tau)\right\} d \tau}{-2 \epsilon+4 g_{n} \mu_{n} B+2 A_{0}+2 \int_{0}^{\frac{1}{2}}\left[J(\tau)-E_{1}^{(0,+)}(\tau)\right] d \tau}=\frac{1+2 m_{-}}{2 m_{4}}, \\
& t_{a}=\frac{2 m_{4} \pi \hbar}{-2 \epsilon+4 g_{n} \mu_{n} B+2 A_{0}+2 \int_{0}^{\frac{1}{2}}\left[J(\tau)-E_{1}^{(0,+)}(\tau)\right] d \tau} .
\end{aligned}
$$

The analytical expression for $E_{1}^{(0,+)}(\tau)$ is shown in Appendix A We find that Eqs. (24) and (25) include two unknown parameters $J_{c}$ and $\tau^{\prime}$, besides the free parameters $m_{+}, m_{-}$, and $m_{4}$. We should choose suitable values for the free parameters that bring about a satisfactory solution. First, we numerically find that both the right-hand sides of Eqs. (24) and (25) are close to $1 / 2$ for $0<J_{c}<\epsilon / 2$ and $0<\tau^{\prime}<1 / 2$. Thus, we choose $m_{+}=m_{-}=0$ and $m_{4}=1$. After this consideration, we numerically solve the simultaneous systems of equation composed of Eqs. (24) and (25) by the Newton-Raphson method. Then, we obtain $t_{a}$ by substituting the value of $m_{4}, J_{c}$, and $\tau^{\prime}$ for Eq. (26). Finally, the remaining parameter $t_{h}$ is determined by Eq. (22).

We show the typical solutions for $\theta_{c z}=\pi$ in Table III The case of $\theta_{c z}=\pi$ is very important, because a CNOT gate is essentially composed of $U_{c z}(\pi)$ and Hadamard gates. We find that if we choose a large value for $J_{c}$, the corresponding values of $\tau^{\prime}$ and $t_{h}$ decrease; the operation time of the controlled $-\mathrm{Z}$ gate becomes short. On the other hand, the value of $t_{a}$ for each $J_{c}$ is almost the same and greatly shorter than $t_{h}$; it suggests that we can safely regard the adiabatic controlling part of the profile of $J(t)$ (i.e., $0 \leq \tau \leq 1 / 2$ and $\tau_{C}-1 / 2 \leq \tau \leq \tau_{C}$ ) as an instantaneous process within the total controlling one.

Let us summarize the above discussion. First, we obtained Eq. (21), which represents the relation between the logical controlled- $\mathrm{Z}$ gate and the physical time evolution, assuming the validity of the adiabatic approximation and the realization of the spin-flip operations by Eq. (21). The result is essentially equivalent to that of Ref. [15], except for the presence of $U_{a d}$. Here, we have shown that $U_{a d}$ is equivalent to the single qubit operation, $\sigma_{1 z} \otimes \sigma_{2 z}$, if we choose a suitable set of the parameters $\left(J_{c}, \tau^{\prime}, t_{a}, t_{h}\right)$. We find that one of the two assumptions, the adiabatic approximation, is valid, be-
TABLE III: Calculated values of $J_{c}, \tau^{\prime}, t_{a}$, and $t_{h}$ with $\theta_{c z}=$ $\pi, m_{+}=0, m_{-}=0$, and $m_{4}=1$.

\begin{tabular}{cccc}
\hline \hline$J_{c} / \epsilon$ & $\tau^{\prime}$ & $t_{a}(\mathrm{n} s)$ & $t_{h}(\mu s)$ \\
\hline 0.1003 & 0.1085 & 5.391 & 33.1 \\
0.1988 & 0.0916 & 5.391 & 12.59 \\
0.01 & 0.2203 & 5.392 & 40.44 \\
\hline \hline
\end{tabular}

cause we choose a value of $J_{c}$ which is far from the level crossing point. Unfortunately, the spin-flip operations by Eq. (21) don't quite work. The main origin of the error in the controlled-Z gates lies in the part relevant to the spin-flip operations (e.g., the Hadamard gate).

\section{SUMMARY}

We have re-investigated the operating schemes of quantum gates associated with universal quantum gates (i.e., $\mathrm{Z}$ rotations, $\mathrm{X}$ rotations, and controlled- $\mathrm{Z}$ gates), for Kane's model in detail. We chose the suitable computational bases out of the eigenstates of $H^{i}(t)$ with fixed time. Our choice is different from those in Refs. 12, 13, 15], in which the electron spin state is assumed to be always down. The bases in the latter case are fragile for the time-evolution due to $H^{i}(t)$. The phase-shift operations (i.e., Z rotations) for the $i$ th qubit are shown to be constructed with an extremely low error probability, adiabatically varying the value of $A_{i}(t)$ with the fixed other parameters: $J(t)=0, A_{j}(t)=A_{0}$, and $B_{a c}=0$. The most important result is that a considerable error for the X rotation in Ref. [15] exists. The physical origin of such an error is the mixing effect between the computational bases and the irrelevant states by $H_{m i x}^{i}$, which is neglected in Ref. [15]. This issue 
emerged from discussion of Eq. (12), not by perturbation but by a rigorous analysis. Finally, the controlled operations in Ref. 15] will not quite work, because the spin-flip operations are used in them. However, it is meaningful to check whether they contain other errors. In particular, we have investigated the controlled $-\mathrm{Z}$ gate, as it is an essential part of the CNOT gate. We have shown that the main origin of the error in the controlled $-\mathrm{Z}$ gate lies in the parts relevant to the spin-flip operations. Accordingly, we will have to discuss recovery of the errors caused by the spin-flip operations.

It is necessary for quantum computation to control quantum systems. In doing this, it is inevitable that some errors occur in the controlling processes, even if we do them carefully. Therefore, it is crucial to make clear the physical origin of the errors and to investigate methods of correcting them. Kane's model is an attractive proposal for quantum computers. In this paper, we have presented the robust quantum computational bases for $H^{i}(t)$ time-evolution, but they do not quite work for the spin-flip operations. We will need to find more suitable computational bases for the operations of quantum gates. In addition, we will have to take account of the modified versions of Kane's model [24, 25].

\section{Acknowledgments}

The authors acknowledge H. Nakazato, K. Yuasa, T. Watanabe, and T. Shinada for valuable discussions. This work is supported partially by a Grant-in-Aid for the COE Research at Waseda University and that for Priority Area B (No.763), MEXT, by a Grant for the 21st Century COE Program at Waseda University, and by a Waseda University Grant for Special Research Projects (No.2004B-872). The authors thank the Yukawa Institute for Theoretical Physics at Kyoto University. Discussions during the YITP workshop YITP-W-0414 on "Chaos and Nonlinear Dynamics in QuantumMechanical and Macroscopic Systems" were useful to the completion of this work.

\section{APPENDIX A: THE INSTANTANEOUS EIGENVALUES OF THE TWO QUBIT HAMILTONIAN}

We show the explicit expressions for the instantaneous eigenvalues of $H_{C}(t)$ at the time $t$ if $A_{1}(t)=A_{2}(t)(\equiv$ $A(t))$. The Hamiltonian $H_{C}(t)$ is a $16 \times 16$ matrix and is decomposed into several submatrices by the system's symmetry (see Eq. (2)). Hereafter, we abbreviate the argument $t$. We only write the eigenvalues relevant to the computation bases:

$$
\begin{aligned}
E_{1}^{(0,1)}=-A & -\frac{1}{2} \sqrt{\xi-c_{1}} \\
& -\frac{1}{2} \sqrt{-\xi-c_{1}+\frac{2 c_{2}}{\sqrt{\xi-c_{1}}}}, \\
E_{2}^{(0,1)}=-A & -\frac{1}{2} \sqrt{\xi-c_{1}} \\
& +\frac{1}{2} \sqrt{-\xi-c_{1}+\frac{2 c_{2}}{\sqrt{\xi-c_{1}}}}, \\
E_{1}^{(-1,-1)}= & -J-\epsilon+2 g_{n} \mu_{n} B \\
& -\sqrt{(\epsilon-2 J)^{2}+4 A^{2}}, \\
E_{2}^{(-1,-1)}= & -J-\epsilon+2 g_{n} \mu_{n} B \\
& +\sqrt{(\epsilon-2 J)^{2}+4 A^{2}}, \\
E_{1}^{(-1,1)}= & J-\epsilon+2 g_{n} \mu_{n} B-\sqrt{\epsilon^{2}+4 A^{2}}, \\
E_{1}^{(-2,1)}= & J-2 \epsilon+4 g_{n} \mu_{n}+2 A .
\end{aligned}
$$

We define $\xi$ in Eqs. (A1) and (A2) as a real root of the following cubic equation:

$$
z^{3}-c_{1} z^{2}-4 c_{3} z+4 c_{3} c_{1}-c_{2}^{2}=0 .
$$

Taking account of Cardano's formula [26], we easily obtain a real root of Eq. A7). The coefficients in Eqs. (A1), (A2), and (A7), $c_{1}, c_{2}$, and $c_{3}$, are given by,

$$
\begin{aligned}
c_{1}= & -6 J^{2}+4 J A-22 A^{2}-4 \epsilon^{2}, \\
c_{2}= & 8 J^{3}-8 A J^{2}+8\left(A^{2}-\epsilon^{2}\right) J+8 \epsilon^{2} A+24 A^{3}, \\
c_{3}= & -3 J^{4}+4 A J^{3}+\left(14 A^{2}+12 \epsilon^{2}\right) J^{2} \\
& \quad+\left(-60 A^{3}+8 \epsilon^{2} A\right) J+12 \epsilon^{2} A^{2}+45 A^{4} .
\end{aligned}
$$

Using Eqs. (19) and (A1) the phases $\alpha_{k}$ and $\beta_{k}$ in Eq.(20) are given as follows:

$$
\begin{aligned}
& \alpha_{1}=\frac{2 t_{a}}{\hbar} \int_{0}^{1 / 2} E_{1}^{(0,1)}(J(\tau)) d \tau \\
& \alpha_{ \pm}=\frac{2 t_{a}}{\hbar} \int_{0}^{1 / 2} E_{1}^{(-1, \pm 1)}(J(\tau)) d \tau \\
& \alpha_{4}=\frac{2 t_{a}}{\hbar} \int_{0}^{1 / 2} E_{1}^{(-2,1)}(J(\tau)) d \tau \\
& \beta_{1}=\frac{t_{h}}{\hbar} E_{1}^{(0,1)}\left(J_{c}\right), \\
& \beta_{ \pm}=\frac{t_{h}}{\hbar} E_{1}^{(-1, \pm 1)}\left(J_{c}\right), \\
& \beta_{4}=\frac{t_{h}}{\hbar} E_{1}^{(-2,1)}\left(J_{c}\right) .
\end{aligned}
$$

\section{APPENDIX B: THE SOLUTION OF THE EIGENVALUE PROBLEM FOR THE SINGLE QUBIT HAMILTONIAN IN THE ROTATING FRAME}

Let us consider the solution for the eigenvalue problem, $H_{\text {rot }}^{i}\left|w_{k}\right\rangle_{i}=\hbar \Omega_{k}^{i}\left|w_{k}\right\rangle_{i}(k=0,1,2,3)$. The eigenvalues 
are given by

$$
\begin{aligned}
\hbar \Omega_{0}^{i}= & -\frac{1}{2} \sqrt{\eta-d_{1}} \\
& -\frac{1}{2} \sqrt{-\eta-d_{1}+\frac{2 d_{2}}{\sqrt{\eta-d_{2}}}} \\
\hbar \Omega_{1}^{i}= & -\frac{1}{2} \sqrt{\eta-d_{1}} \\
& +\frac{1}{2} \sqrt{-\eta-d_{1}+\frac{2 d_{2}}{\sqrt{\eta-d_{2}}}} \\
\hbar \Omega_{2}^{i}= & \frac{1}{2} \sqrt{\eta-d_{1}} \\
& -\frac{1}{2} \sqrt{-\eta-d_{1}-\frac{2 d_{2}}{\sqrt{\eta-d_{2}}}} \\
\hbar \Omega_{3}^{i}= & \frac{1}{2} \sqrt{\eta-d_{1}} \\
& +\frac{1}{2} \sqrt{-\eta-d_{1}-\frac{2 d_{2}}{\sqrt{\eta-d_{2}}}}
\end{aligned}
$$

We define $\eta$ in Eqs. (B1)-(B4) as a real root of the following cubic equation:

$$
z^{3}-d_{1} z^{2}-4 d_{3} z+4 d_{1} d_{3}-d_{2}^{2}=0
$$

We easily find a real root of Eq. B5 through Cardano's formula 26], as in Appendix A The coefficients, $d_{1}, d_{2}$, and $d_{3}$ in Eqs. (B1) - B5 , are given by

$$
\begin{aligned}
& d_{1}=E_{0}^{i} E_{2}^{i}-\left(E_{0}^{i}+E_{2}^{i}\right)^{2}+\left(E_{1}^{i}-\hbar \omega_{a c}\right)\left(E_{3}^{i}+\hbar \omega_{a c}\right)-2\left(\mu_{B} B_{a c}\right)^{2}-2\left(g_{n} \mu_{n} B_{a c}\right)^{2}, \\
& d_{2}=\left(E_{0}^{i}+E_{2}^{i}\right)\left[E_{0}^{i} E_{2}^{i}-\left(E_{1}^{i}-\hbar \omega_{a c}\right)\left(E_{3}^{i}+\hbar \omega_{a c}\right)\right] \\
& \quad+4 g_{n} \mu_{n} \mu_{B} B_{a c}^{2} A, \\
& d_{3}=E_{0}^{i} E_{2}^{i}\left(E_{1}^{i}-\hbar \omega_{a c}\right)\left(E_{3}^{i}+\hbar \omega_{a c}\right)+\left(\mu_{B}^{2}-g_{n}^{2} \mu_{n}^{2}\right)^{2} B_{a c}^{2} \\
&-\left(E_{1}^{i}-\hbar \omega_{a c}\right)\left(E_{0}^{i} \nu_{-\theta}^{2}+E_{2}^{i} \mu_{-\theta}^{2}\right) B_{a c}^{2} \\
&-\left(E_{3}^{i}+\hbar \omega_{a c}\right)\left(E_{0}^{i} \mu_{\theta}^{2}+E_{2}^{i} \nu_{\theta}^{2}\right) B_{a c}^{2} .
\end{aligned}
$$

The eigenvector $\left|w_{k}\right\rangle_{i}$ associated with the eigenvalue $\hbar \Omega_{k}^{i}$ is given by

$$
\begin{aligned}
\left|w_{k}\right\rangle_{i}= & \mathcal{N}\left[\left(-\frac{\nu_{-\theta}}{\mu_{-\theta}} \frac{E_{0}^{i}-\hbar \Omega_{k}^{i}}{\nu_{\theta} B_{a c}}-\frac{E_{2}^{i}-\hbar \Omega_{k}^{i}}{\mu_{\theta} B_{a c}}\right)\left|u_{0}(A)\right\rangle_{i}\right. \\
& +\left(\frac{\mu_{-\theta}}{\nu_{\theta}}-\frac{\nu_{-\theta}}{\mu_{\theta}}-\frac{E_{3}^{i}+\hbar \omega_{a c}-\hbar \Omega_{k}^{i}}{\mu_{-\theta} B_{a c}} \frac{E_{0}^{i}-\hbar \Omega_{k}^{i}}{\nu_{\theta} B_{a c}}\right)\left|u_{1}(A)\right\rangle_{i} \\
& +\left(\frac{E_{3}^{i}+\hbar \Omega_{a c}-\hbar \Omega_{k}^{i}}{\mu_{-\theta} B_{a c}} \frac{E_{2}^{i}-\hbar \Omega_{k}^{i}}{\mu_{\theta} B_{a c}}+\frac{\nu_{-\theta}}{\nu_{\theta}}-\frac{\nu_{-\theta}^{2}}{\mu_{\theta} \mu_{-\theta}}\right)\left|u_{2}(A)\right\rangle_{i} \\
& \left.+\left(\frac{E_{3}^{i}+\hbar \omega_{a c}-\hbar \Omega_{k}^{i}}{\mu_{\theta} B_{a c}} \frac{E_{2}^{i}-\hbar \Omega_{k}^{i}}{\mu_{-\theta} B_{a c}} \frac{E_{0}^{i}-\hbar \Omega_{k}^{i}}{\nu_{\theta}}-\frac{\nu_{-\theta}^{2}}{\mu_{\theta} \mu_{-\theta}} \frac{E_{0}^{i}-\hbar \Omega_{k}^{i}}{\nu_{\theta}}-\frac{\mu_{-\theta}}{\mu_{\theta}} \frac{E_{2}^{i}-\hbar \Omega_{k}^{i}}{\nu_{\theta}}\right)\left|u_{3}(A)\right\rangle_{i}\right],
\end{aligned}
$$

where $\mathcal{N}$ is a normalization factor.

[1] E. Knill, I. Chuang, and R. Laflamme, Phys. Rev. A 57, 3348 (1998).

[2] N. A. Gershenfeld and I. L. Chuang, Science 275, 350 (1997).

[3] D. G. Cory, A. F. Fahmy, and T. F. Havel, Proc. Natl. Acad. Sci. USA 94, 1634 (1997).

[4] L. M. K. Vandersypen, M. Steffen, G. Breyta, C. S. Yannoni, R. Cleve, and I. L. Chuang, Phys. Rev. Letts. 85, 5452 (2000).

[5] B. E. Kane, Nature, 393, 133 (1998).

[6] T. D. Ladd, J. R. Goldman, F. Yamaguchi, Y. Yamamoto, E. Abe, and K. M. Itoh, Phys. Rev. Letts. 89, 017901 (2002).

[7] D. G. Cory et al., Fortschr. Phys. 48, 875 (2000).

[8] T. Shinada, A. Ishikawa, C. Hinoshita, M. Koh, and I. Ohdomari, Jpn. J. Appl. Phys. 39, L265 (2000); T. Shinada, H. Koyama, C. Hinoshita, K. Imamura, and I.
Ohdomari, Jpn. J. Appl. Phys. 41, L287 (2002).

[9] O. Astafiev, Y. A. Pashkin, T. Yamamoto, Y. Nakamura, and J. S. Tsai, Phys. Rev. B 69, 180507(R) (2004).

[10] J. M. Elzerman, R. Hanson, L. H. M. van Beveren, B. Witkamp, L. M. K. Vandersypen, and L. P. Kouwenhoven, Nature 430, 431 (2004).

[11] M. Xiao, I. Martin, E. Yablonovitch, and H. W. Jiang, Nature 430, 435 (2004).

[12] C. Wellard, L. C. L. Hollenberg, and H. C. Pauli, Phys. Rev. A 65, 032303 (2002).

[13] A. G. Fowler, C. J. Wellard, and L. C. L. Hollenberg, Phys. Rev. A 67, 012301 (2003).

[14] A. Galindo and M. A. Martín-Delgado, Rev. Mod. Phys., 74, 347 (2002).

[15] C. D. Hill and H. -S. Goan, Phys. Rev. A 68, 012321 (2003); Phys. Rev. A 70, 022310 (2004).

[16] M. A. Nielsen and I. L. Chuang, Quantum Computation 
and Quantum Information (Cambridge University Press, Cambridge, England, 2000).

[17] G. P. Berman. D. K. Campbell, G. D. Doolen, and K. E. Nagaev, J. Phys.: Condens. Matter 12, 2945 (2000).

[18] T. Kato, J. Phys. Soc. Jpn. 5, 435 (1950).

[19] A. Messiah, Quantum Mechanics (North-Holland, Amsterdam, 1965), Vol. 2.

[20] R. A. Horn and C. R. Johnson, Matrix Analysis (Cambridge University Press, Cambridge, England, 1985).

[21] A. Abragam, The Principles of Nuclear Magnetism (Oxford University Press, London, 1961).
[22] C. P. Slichter, Principles of Magnetic Resonance, 2nd ed. (Springer, Berlin, 1978).

[23] J. Preskill, Proc. R. Soc. London, Ser A 454, 385 (1998).

[24] A. J. Skinner, M. E. Davenport, and B. E. Kane, Phys. Rev. Letts. 90, 087901 (2003).

[25] C. D. Hill, L. C. L. Hollenberg, A. G. Fowler, C. J. Wellard, A. D. Greentree, and H.-S. Goan, e-print quant-ph/0411104 (2004).

[26] J.-P. Tignol, Galois' Theory of Algebraic Equations (World Scientific, Singapore, 2001). 